\title{
Opportunistic Communications in an Orthogonal Multiaccess Relay Channel
}

\author{
Lalitha Sankar \\ WINLAB, Dept. of ECE \\ Rutgers University \\ North Brunswick, NJ 08902 \\ lalitha@winlab.rutgers.edu
}

\author{
Yingbin Liang, H. Vincent Poor \\ Dept. of Electrical Engineering \\ Princeton University \\ Princeton, NJ 08544 \\ \{yingbinl,poor\}@ princeton.edu
}

\author{
Narayan Mandayam \\ WINLAB, Dept. of ECE \\ Rutgers University \\ North Brunswick, NJ 08902 \\ narayan@winlab.rutgers.edu
}

\begin{abstract}
The problem of resource allocation is studied for a two-user fading orthogonal multiaccess relay channel (MARC) where both users (sources) communicate with a destination in the presence of a relay. A half-duplex relay is considered that transmits on a channel orthogonal to that used by the sources. The instantaneous fading state between every transmit-receive pair in this network is assumed to be known at both the transmitter and receiver. Under an average power constraint at each source and the relay, the sum-rate for the achievable strategy of decode-and-forward (DF) is maximized over all power allocations (policies) at the sources and relay. It is shown that the sum-rate maximizing policy exploits the multiuser fading diversity to reveal the optimality of opportunistic channel use by each user. A geometric interpretation of the optimal power policy is also presented.
\end{abstract}

\section{INTRODUCTION}

The multiaccess relay channel (MARC) is a network in which several users (source nodes) communicate with a single destination in the presence of a relay [1]. The MARC is a model for relay-based cooperation in a multiuser network where the users have limited power and processing capabilities or need tangible incentives to cooperate. We model a MARC with a half-duplex relay as an orthogonal MARC where the relay transmits on a channel orthogonal to that used by the sources (see [2], [3]). The coding strategies developed for the relay channel [4] extend readily to the MARC [3]. For example, the strategy of [4, Theorem 1], now often called decodeand-forward (DF), has a relay that decodes user messages before forwarding them to the destination [5], [6]. Similarly, the strategy in [4, Theorem 6], now often called compressand-forward $(\mathrm{CF})$, has the relay quantize its output symbols and transmit the resulting quantized bits to the destination [3].

We study the problem of resource allocation in a twouser ergodic fading orthogonal MARC employing DF under the assumption that the instantaneous fading state between each transmit-receive pair in this network is known at both the transmitter and receiver. Resource allocation for a singleuser ergodic fading orthogonal relay channel employing DF and subject to an average power constraint at the source and

This research was supported in part by the US National Science Foundation under Grants ANI-03-38807, CCR-04-29724 and CNS-06-25637. relay is studied in [2] (see also [7]). The authors formulate the problem as a max-min optimization. They draw parallels with the classic minimax optimization in hypothesis testing to show that the optimal resource allocation achieves one of three solutions depending on the joint fading statistics. The orthogonal MARC studied here is a multiaccess generalization of the orthogonal relay channel in [2]; however, the optimal policies developed in [2] do not extend readily to maximize the sum-rate of the MARC. For a two-user MARC, we show that the DF sum-rate belongs to one of five disjoint cases or lies on the boundary of any two of them. Our results reveal two interesting observations: 1) analogously to a classic fading multiaccess channel [8], [9], the sum-rate optimal policy for each case exploits the multiuser fading diversity to opportunistically schedule users; 2) however, these optimal policies are not necessarily water-filling solutions. Finally, we present a geometric interpretation for each case to highlight the effects of node topology in the analysis of multi-terminal networks.

The paper is organized as follows. In Section П, we model the orthogonal MARC with Gaussian noise and fading. In Section [II we present the rate region and determine the power policies that maximize the DF sum-rate. Finally, we conclude in Section IV

\section{Channel Model AND PREliminaries}

A two-user MARC consists of two source nodes numbered 1 and 2, a relay node $r$, and a destination node $d$. We write $\mathcal{K}=\{1,2\}$ to denote the set of sources, $\mathcal{T}=\mathcal{K} \cup\{r\}$ to denote the set of transmitters, and $\mathcal{D}=\{r, d\}$ to denote the set of receivers. In an orthogonal MARC, the sources transmit to the relay and destination on one channel, say channel 1, while the half-duplex relay transmits to the destination on an orthogonal channel 2 as shown in Fig. 1. A fraction $\theta$ of the total bandwidth resource is allocated to channel 1 while the remaining fraction $\bar{\theta}=1-\theta$ is allocated to channel 2. In the fraction $\theta$, the source $k$ transmits the signal $X_{k}$ while the relay and the destination receive $Y_{r}$ and $Y_{d, 1}$ respectively. In the fraction $\bar{\theta}$, the relay transmits $X_{r}$ and the destination receives 


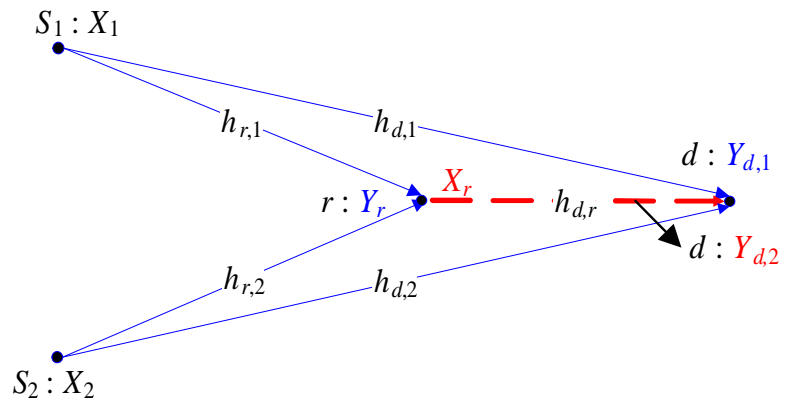

Fig. 1. A two-user orthogonal MARC.

$Y_{d, 2}$. In each time symbol (channel use), we then have

$$
\begin{aligned}
Y_{r} & =h_{r, 1} X_{1}+h_{r, 2} X_{2}+Z_{r} \\
Y_{d, 1} & =h_{d, 1} X_{1}+h_{d, 2} X_{2}+Z_{d, 1} \\
Y_{d, 2} & =h_{d, r} X_{r}+Z_{d, 2}
\end{aligned}
$$

where $Z_{r}, Z_{d, 1}, Z_{d, 2}$ are independent circularly symmetric complex Gaussian noise random variables with zero means and unit variances. We write $\underline{h}$ to denote the vector of fading gains, $h_{k, m}$, for all $k \in \mathcal{D}$ and $m \in \mathcal{T}, k \neq m$, such that $\underline{h}$ is a realization for a given channel use of a jointly stationary and ergodic vector fading process $\underline{H}$. We assume that the fraction $\theta$ is fixed a priori and is known at all nodes.

Over $n$ uses of the channel, the source and relay tranmissions are constrained in power according to

$$
\sum_{i=1}^{n} \mathbb{E}\left(\left|X_{k i}\right|^{2}\right) \leq n \bar{P}_{k} \text { for all } k \in \mathcal{T} .
$$

Since the sources and relay know the fading states of the links on which they transmit, they can allocate their transmitted signal power according to the channel state information. We write $P_{k}(\underline{h})$ to denote the power allocated as a function of the channel states $\underline{h}$ at the $k^{t h}$ transmitter, for all $k \in \mathcal{T}$. For an ergodic fading channel, (4) then simplifies to

$$
\mathbb{E}\left(P_{k}(\underline{h})\right) \leq \bar{P}_{k} \text { for all } k \in \mathcal{T}
$$

where the expectation in (5) is over the joint distribution $\underline{H}$. We write $\underline{P}(\underline{h})$ to denote a vector of power allocations with entries $P_{k}(\underline{h})$ for all $k \in \mathcal{T}$, and define $\mathcal{P}$ to be the set of all $\underline{P}(\underline{h})$ whose entries satisfy (5). For ease of notation, we henceforth omit the functional dependence of $\underline{P}$ on $\underline{h}$. We use the notation $C(x)=\log (1+x)$ where the logarithm is to the base $2,(x)^{+}=\max (x, 0)$, and write $R_{\mathcal{S}}=\sum_{k \in \mathcal{S}} R_{k}$ for any $\mathcal{S} \subseteq \mathcal{K}$.

\section{Sum-Rate Optimal Power Policy}

The DF rate region for a MARC with fixed channel gains and a full-duplex relay is developed in [5, Appendix A] (see also [6]). For a half-duplex MARC with a fixed $\underline{h}$ and a fixed fraction $\theta$, the DF rate region includes an additional conditioning on the half-duplex modes of the relay [3] and is the set of all rate pairs $\left(R_{1}, R_{2}\right)$ that satisfy

$$
R_{k} \leq \min \left\{\begin{array}{c}
\theta C\left(\frac{\left|h_{d, k}\right|^{2} P_{k}}{\theta}\right)+\bar{\theta} C\left(\frac{\left|h_{d, r}\right|^{2} P_{r}}{\bar{\theta}}\right), \\
\theta C\left(\frac{\left|h_{r, k}\right|^{2} P_{k}}{\theta}\right)
\end{array}\right\}, k=1,2
$$

and

$$
R_{1}+R_{2} \leq \min \left\{\begin{array}{c}
\theta C\left(\sum_{k=1}^{2} \frac{\left|h_{d, k}\right|^{2} P_{k}}{\theta}\right)+\bar{\theta} C\left(\frac{\left|h_{d, r}\right|^{2} P_{r}}{\bar{\theta}}\right), \\
\theta C\left(\sum_{k=1}^{2} \frac{\left|h_{r, k}\right|^{2} P_{k}}{\theta}\right)
\end{array}\right\} .
$$

For a stationary and ergodic vector process $\underline{H}$, the channel in (1)-(3) can be modeled as a set of parallel Gaussian orthogonal MARCs, one for each fading instantiation $\underline{h}$. For a fixed $\underline{P}$, the DF rate bounds for this ergodic fading channel are obtained by averaging the bounds in (6) and (7) over all channel realizations. The DF rate region, $\mathcal{R}_{D F}$, achieved over all $\underline{P} \in \mathcal{P}$, is given by the following theorem.

Theorem 1: The DF rate region, $\mathcal{R}_{D F}$, achieved over an ergodic fading orthogonal Gaussian MARC is

$$
\mathcal{R}_{D F}=\bigcup_{\underline{P} \in \mathcal{P}}\left\{\mathcal{R}_{r}(\underline{P}) \cap \mathcal{R}_{d}(\underline{P})\right\}
$$

where, for all $\mathcal{S} \subseteq \mathcal{K}$, we have

$$
\mathcal{R}_{r}(\underline{P})=\left\{\left(R_{1}, R_{2}\right): R_{\mathcal{S}} \leq \theta \mathbb{E} C\left(\frac{\sum_{k \in \mathcal{S}}\left|h_{r, k}\right|^{2} P_{k}}{\theta}\right)\right\}
$$

and

$$
\mathcal{R}_{d}(\underline{P})=\left\{\begin{array}{c}
\left(R_{1}, R_{2}\right): R_{\mathcal{S}} \leq \theta \mathbb{E} C\left(\frac{\sum_{k \in \mathcal{S}}\left|h_{d, k}\right|^{2} P_{k}}{\theta}\right) \\
+\bar{\theta} \mathbb{E} C\left(\frac{\left|h_{d, r}\right|^{2} P_{r}}{\bar{\theta}}\right)
\end{array}\right\}
$$

Remark 2: The rate region $\mathcal{R}_{D F}$ is convex. This follows from the convexity of the set $\mathcal{P}$ and the concavity of the log function.

The region $\mathcal{R}_{D F}$ in $(8)$ is a union of the intersections of the regions $\mathcal{R}_{r}(\underline{P})$ and $\mathcal{R}_{d}(\underline{P})$ achieved at the relay and destination respectively, where the union is over all $\underline{P} \in \mathcal{P}$. Since $\mathcal{R}_{D F}$ is convex, each point on the boundary of $\mathcal{R}_{D F}$ is obtained by maximizing the weighted sum $\mu_{1} R_{1}+\mu_{2} R_{2}$ over all $\underline{P} \in \mathcal{P}$, and for all $\mu_{1}>0, \mu_{2}>0$. Specifically, we determine the optimal policy $\underline{P}^{*}$ that maximizes the sumrate $R_{1}+R_{2}$ when $\mu_{1}=\mu_{2}=1$. Observe from $(8)$ that every point on the boundary of $\mathcal{R}_{D F}$ results from the intersection of $\mathcal{R}_{r}(\underline{P})$ and $\mathcal{R}_{d}(\underline{P})$ for some $\underline{P}$. In Figs. 2 and 3 we illustrate the five possible choices for the sum-rate resulting from such an intersection. Case 1 and case 2 result when no rate pair on the sum-rate plane achieved at one receiver lies within or on the boundary of the rate region achieved at the other receiver (see Fig. 2). On the other hand, cases $3 a, 3 b$, and $3 c$ result 


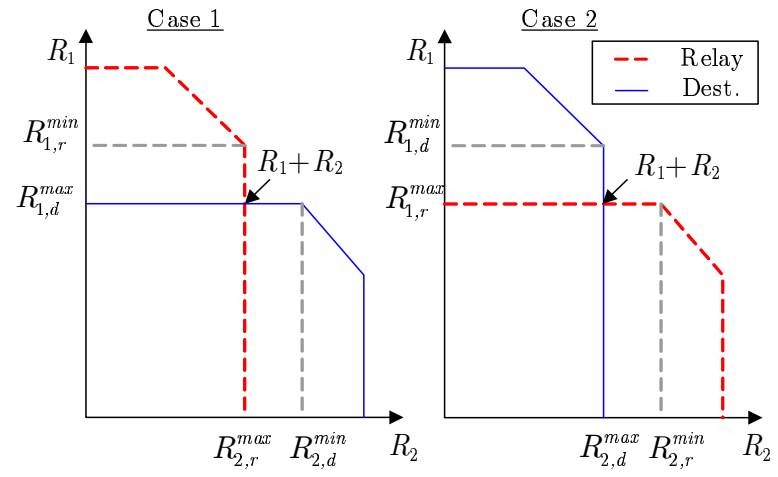

Fig. 2. Rate region and sum-rate for case 1 and case 2 .

when there is more than one such rate pair as shown in Fig. (3) Observe that case $3 c$ corresponds to a boundary case where the sum-rate planes overlap. We also consider six boundary cases where there is exactly one such rate pair that serves as a transition between case 1 or 2 and one of cases $3 a, 3 b$, or $3 c$. An example of a boundary case for case 1 and case $3 a$ is shown in Fig. 3. We write $\mathcal{B}_{m} \subseteq \mathcal{P}$ to denote the set of $\underline{P}$ that achieve case $i, i=1,2,3 a, 3 b, 3 c$ and $\mathcal{B}_{l, n}, l=1,2$, $\underline{n}=3 a, 3 b, 3 c$ to denote the set of $\underline{P}$ satisfying each boundary case. We show in the sequel that the optimization is simplified considerably when the conditions for each case are defined such that the sets $\mathcal{B}_{i}$ and $\mathcal{B}_{l, n}$ are disjoint for all $i, l, n$, and thus, are either open or half-open sets such that no two sets share a boundary. Finally, we observe that cases 1 and 2 do not share a boundary since such a transition (see Fig. 2) requires passing through case $3 a$ or $3 b$ or $3 c$.

To determine the optimal $\underline{P}^{*}$, we first define the conditions for each case and determine the policy $\underline{P}^{(i)}$ or $\underline{P}^{(l, n)}$ maximizing the sum-rate for case $i$ or the boundary case $(l, n)$. We collect the six boundary cases as the last case. The optimal policy for each case is determined using Lagrange multipliers and the Karush-Kuhn-Tucker (KKT) conditions [10, 5.5.3].

Case 1: This case occurs when the power policy $\underline{P} \in \mathcal{B}_{1}$ achieves the relay and destination regions shown in Fig 2 , The maximum sum-rate achieved in this case is

$$
\max _{\underline{P} \in \mathcal{B}_{1}}\left(R_{1, d}^{\max }(\underline{P})+R_{2, r}^{\max }(\underline{P})\right)
$$

where $R_{k, m}^{\max }$ is the maximum rate achieved by user $k$ at receiver $m \in \mathcal{D}$ in (9) and (10). The open set $\mathcal{B}_{1}$ contains all $\underline{P}$ that satisfy

$$
R_{1, d}^{\max }(\underline{P})<R_{1, r}^{\min }(\underline{P}) \text { and } R_{2, r}^{\max }(\underline{P})<R_{2, d}^{\min }(\underline{P})
$$

where $R_{k, m}^{\min }$ is the rate achieved by user $k$ when it is the first user to be successively decoded at a sum-rate corner point achieved at receiver $m$. Since $\mathcal{B}_{1}$ is not known a priori, we determine the optimal $\underline{P}^{(1)}$ maximizing $R_{1}+R_{2}$ in (11) over $\mathcal{P}$. Expanding (11) using (9) and (10) and applying the Lagrange multiplier rule and the KKT conditions, we obtain

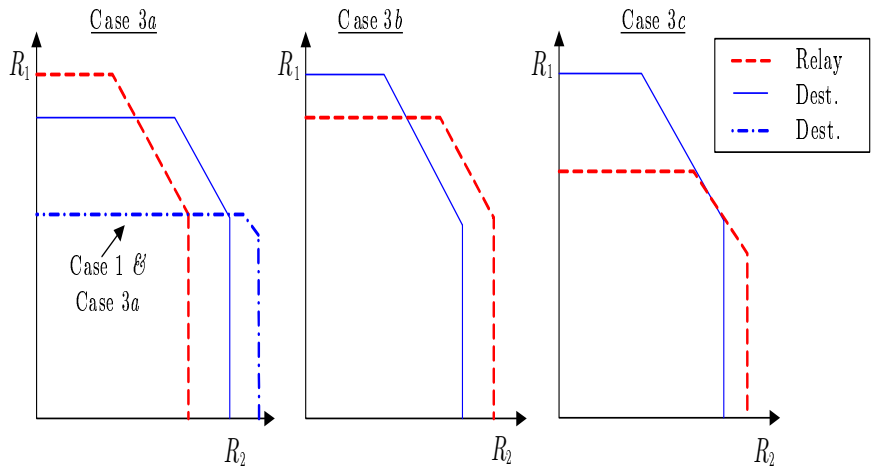

Fig. 3. Rate region and sum-rate for cases $3 a, 3 b$, and $3 c$.

$P_{k}^{(1)}$ and $P_{r}^{(1)}$ as

$$
P_{k}^{(1)}=\left(\frac{\theta}{\nu_{k} \ln 2}-\frac{\theta}{\left|h_{m, k}\right|^{2}}\right)^{+} \quad(k, m)=(1, d),(2, r)
$$

and

$$
P_{r}^{(1)}=\left(\frac{\bar{\theta}}{\nu_{r} \ln 2}-\frac{\bar{\theta}}{\left|h_{d, r}\right|^{2}}\right)^{+}
$$

where the water-filling level $\nu_{k}, k=1,2, r$, is determined from (5). To ensure that case 1 occurs, we require that $\underline{P}^{(1)} \in \mathcal{B}_{1}$, i.e., (13) and (14) satisfy (12). Then, the concavity of the rate functions in (9) and (10) suffices to show that $\underline{P}^{(1)}$ in $(13)$ and (14) maximizes (11). On the other hand, when $\underline{P}^{(1)} \notin \mathcal{B}_{1}$, we show that $R_{1}+R_{2}$ achieves its maximum outside $\mathcal{B}_{1}$. Note that the expression for $R_{1}+R_{2}$ for the other cases is not the same as that in (11). The proof follows from the fact that $R_{1}+R_{2}$ in (11) is a concave function of $\underline{P}$ for all $\underline{P} \in \mathcal{P}$. Thus, when $\underline{P}^{(1)} \notin \mathcal{B}_{1}$, for every $\underline{P} \in \mathcal{B}_{1}$ there exists a $\underline{P^{\prime}} \in \mathcal{B}_{1}$ with a larger sum-rate. Combining this with the fact that the sumrate expressions are continuous while transitioning from one case to another at the boundary of the open set $\mathcal{B}_{1}$, ensures that the maximum sum-rate is achieved by some $\underline{P} \notin \mathcal{B}_{1}$. We remark that similar arguments also apply to the remaining cases, and will be omitted for brevity. Finally, we remark that (12) models a network geometry in which the destination and source 1 are physically proximal, i.e., they form a cluster, and the relay and source 2 form another cluster.

Case 2: The maximum sum-rate achieved for this case is

$$
\max _{\underline{P} \in \mathcal{B}_{2}}\left(R_{1, r}^{\max }(\underline{P})+R_{2, d}^{\max }(\underline{P})\right)
$$

where $\mathcal{B}_{2}$ contains all $\underline{P}$ that satisfy

$$
R_{1, r}^{\max }(\underline{P})<R_{1, d}^{\min }(\underline{P}) \text { and } R_{2, d}^{\max }(\underline{P})<R_{2, r}^{\min }(\underline{P}) .
$$

As in case 1, we can show that (15) is maximized by setting the optimal $P_{k}^{(2)}$ and $P_{r}^{(2)}$ to the expressions in (13) and (14) respectively with $(k, m)=(1, r),(2, d)$ provided the resulting $\underline{P}^{(2)}$ satisfies (16). Finally, we remark that the conditions in (16) model a network geometry in which the destination and source 2 form one cluster while the relay and source 1 form another cluster. 
Case 3: Consider the cases $3 a, 3 b$, and $3 c$ shown in Fig. 3 The sum-rate optimization for all three cases simplifies to

$$
\max _{\underline{P} \in \mathcal{B}_{3}} \min \left(\left(R_{\mathcal{K}}\right)_{r},\left(R_{\mathcal{K}}\right)_{d}\right)
$$

where $\left(R_{\mathcal{K}}\right)_{r}$ and $\left(R_{\mathcal{K}}\right)_{d}$ are the mutual information expressions in (9) and (10) respectively for $\mathcal{S}=\mathcal{K}$, and $\mathcal{B}_{3}$ consists of $\underline{P}$ that do not satisfy (12) and (16) either as strict inequalities or with equality. We write $\mathcal{B}_{3}=\mathcal{B}_{3 a} \cup \mathcal{B}_{3 b} \cup \mathcal{B}_{3 c}$, where $\mathcal{B}_{i}$, $i=3 a, 3 b, 3 c$ is defined for case $i$ below. The optimization in (17) is a multiuser generalization of the single-user max-min problem studied in [2]. Extending the known general solution to (17), the optimal policy $\underline{P}^{(3)}$ for this case satisfies one of following three conditions

$$
\begin{array}{ll}
\text { Case 3a: } & \left.\left(R_{\mathcal{K}}\right)_{r}\right|_{\underline{P}^{(3)}}<\left.\left(R_{\mathcal{K}}\right)_{d}\right|_{\underline{P}^{(3)}} \\
\text { Case 3b: } & \left.\left(R_{\mathcal{K}}\right)_{r}\right|_{\underline{P}^{(3)}}>\left.\left(R_{\mathcal{K}}\right)_{d}\right|_{\underline{P}^{(3)}} \\
\text { Case 3c: } & \left.\left(R_{\mathcal{K}}\right)_{r}\right|_{\underline{P}^{(3)}}=\left.\left(R_{\mathcal{K}}\right)_{d}\right|_{\underline{P}^{(3)}} .
\end{array}
$$

We proceed to study cases $3 a, 3 b$, and $3 c$ in detail.

Case 3a: Maximizing $\left(R_{\mathcal{K}}\right)_{r}$ subject to (5) results in the KKT conditions

$$
f_{k} \leq \nu_{k} \ln 2, \quad k=1,2
$$

where

$$
f_{k}=\left|h_{r, k}\right|^{2} /\left(1+\sum_{k=1}^{2} \frac{\left|h_{r, k}\right|^{2} P_{k}}{\theta}\right) .
$$

For those channel states in which the two users do not see the same scaled fading gains at the relay, i.e., $\left|h_{r, 1}\right| / \nu_{1} \neq$ $\left|h_{r, 2}\right| / \nu_{2}$, 21) reduces, for all $k, j \in \mathcal{K}, k \neq j$, and $i=3 a$, to

$$
P_{k}^{(i)}=\left\{\begin{array}{cc}
\left(\frac{\theta}{\nu_{k} \ln 2}-\frac{\theta}{\left|h_{r, k}\right|^{2}}\right)^{+} & f_{k}>f_{j} \frac{\nu_{k}}{\nu_{j}}, j \neq k \\
0 & \text { o.w. }
\end{array} .\right.
$$

Observe that the optimal $P_{k}^{(3 a)}$ in 23) is an opportunistic water-filling solution that exploits the fading diversity in a multiaccess channel with the relay as the receiver. The optimal policy at the relay $P_{r}^{(3 a)}$ is given by $(14)$. For $\underline{P}^{(3 a)} \in \mathcal{B}_{3}$, the requirement of satisfying (18), i.e., $\underline{P}^{(3 a)} \in \mathcal{B}_{3 a}$, simplifies to a threshold condition $\bar{P}_{r}>P_{u}\left(\bar{P}_{1}, \bar{P}_{2}\right)$ where $\bar{P}_{k}, k \in \mathcal{T}$, is defined in (5) and the threshold $P_{u}\left(\bar{P}_{1}, \bar{P}_{2}\right)$ is obtained by setting (18) to an equality. When $\underline{P}^{(3 a)} \in \mathcal{B}_{3}$ but $\underline{P}^{(3 a)} \notin \mathcal{B}_{3 a}$, $R_{1}+R_{2}$ is maximized by either case $3 b$ or case $3 c$. For $\underline{P}^{(3 a)}$ $\notin \mathcal{B}_{3}$, as argued in case 1 the sum-rate is not maximized by any $\underline{P} \in \mathcal{B}_{3}$. Finally, the condition in $(18)$ suggests a geometry where the sources and destination are clustered.

Case $3 b$ : The KKT conditions and optimal $P_{k}^{(3 b)}$ for this case maximize $\left(R_{\mathcal{K}}\right)_{d}$ and are given by (21) and (23) respectively with the subscript ' $r$ ' in (22) changed to ' $d$ ' for all $k$ and $i=3 b$. Further, (21) and 22) also hold for the relay node, $k=r$, and simplifies to the water-filling solution in (14). Thus, as in case $3 a$, it is optimal to time-duplex the users except that this is now based on their scaled fading gains to the destination. For $\underline{P}^{(3 b)} \in \mathcal{B}_{3}$, satisfying $\left[19\right.$, i.e., $\underline{P}^{(3 b)}$ $\in \mathcal{B}_{3 b}$, reduces to satisfying the threshold condition $\bar{P}_{r}<$ $P_{l}\left(\bar{P}_{1}, \bar{P}_{2}\right)$ where $P_{l}\left(\bar{P}_{1}, \bar{P}_{2}\right)$ is determined by setting 19 to an equality. Finally, 19] implies a geometry in which the sources are clustered closer to the relay than to the destination. Case $3 c$ (equalizer policy): Maximizing $\left(R_{\mathcal{K}}\right)_{r}$ over all $\underline{P}$, subject to (20) gives $\underline{P}^{(3 c)}$. This case occurs when $\underline{P}^{(3 c)} \in$ $\mathcal{B}_{3 c}$, i.e. $P_{l}\left(\bar{P}_{1}, \bar{P}_{2}\right) \leq \bar{P}_{r} \leq P_{u}\left(\bar{P}_{1}, \bar{P}_{2}\right)$. The resulting KKT conditions take the form in (21) such that for $k=1,2$,

$$
f_{k}=\frac{(1-\alpha)\left|h_{r, k}\right|^{2}}{1+\sum_{j=1}^{2}\left|h_{r, j}\right|^{2} P_{j} / \theta}+\frac{\alpha\left|h_{d, k}\right|^{2}}{1+\sum_{j=1}^{2}\left|h_{d, j}\right|^{2} P_{j} / \theta} .
$$

For $k=r, f_{r}$ is obtained by replacing $h_{r, k}$ in (22) by $h_{d, r}$ and scaling by $\alpha$. The Lagrange multiplier $\alpha$ accounts for the boundary condition in (20) and is computed by evaluating (20) at $\underline{P}^{(3 c)}$. The relay's optimal policy simplifies to the waterfilling solution in (14) with the first term scaled by $\alpha$. For $\left|h_{m, 1}\right| / \nu_{1} \neq\left|h_{m, 2}\right| / \nu_{2}, m=r$ or $d$, (21) simplifies, for $f_{k}$ in (24) and for all $k, j \in \mathcal{K}, k \neq j$, and $i=3 c$ as

$$
P_{k}^{(i)}=\left\{\begin{array}{cc}
\left(\begin{array}{cc}
\text { root of }\left.f_{k}\right|_{P_{j}=0}
\end{array}\right)^{+} & f_{k}>f_{j} \\
0 & \text { o.w. }
\end{array}\right.
$$

From (25), we see that user $k, k=1,2$, transmits opportunistically over those channel states where a function $f_{k}$ of its fading gains and power is larger than that of the other user. Note that the optimal policy $P_{k}^{(3 c)}$ in $(25)$ is no longer a waterfilling solution at the two sources. Finally, we remark that this boundary case occurs for a range of geometries that transition from the clustered geometry of case $3 a$ to that of case $3 b$.

Case 4: Boundary Cases: We now consider the six boundary cases and summarize the optimal $\underline{P}^{(l, n)}, l=1,2, n=3 a$, $3 b, 3 c$, for each case. As with case $3 c$, we observe that the geometries for these boundary cases also straddle the clustered geometries of the two cases involved.

Case 1 and Case 3a: The sum-rate optimization for this case simplifies to

$$
\max _{P \in \mathcal{B}_{1,3 a}}\left(R_{\mathcal{K}}\right)_{r} \text { s.t. }\left(R_{\mathcal{K}}\right)_{r}=R_{1, d}^{\max }+R_{2, r}^{\max }
$$

where $\mathcal{B}_{1,3 a}$ is the set of all $\underline{P}$ that satisfy $(18)$, (26), and one of the inequalities in (12) (see Fig. 3). Note that from (9), we can write $\left(R_{\mathcal{K}}\right)_{r}=R_{1, r}^{\min }+R_{2, r}^{\max }$ thus simplifying the condition in (26) to $R_{1, r}^{\min }=R_{1, d}^{\max }$ as shown in Fig. 3 . As before, we obtain the KKT conditions in (21) for $k=1,2$, $(k, m)=(1, d),(2, r)$, and

$$
f_{k}=\frac{(1-\alpha)\left|h_{r, k}\right|^{2}}{1+\sum_{j=1}^{2} \frac{\left|h_{r, j}\right|^{2} P_{j}}{\theta}}+\frac{\alpha\left|h_{m, k}\right|^{2}}{1+\frac{\left|h_{m, k}\right|^{2} P_{k}}{\theta}}
$$

where $\alpha$ is the Lagrange multiplier satisfying the boundary condition in 26. When $\left|h_{m, 1}\right| / \nu_{1} \neq\left|h_{m, 2}\right| / \nu_{2}, m=r$ or $d$, (21) simplifies $P_{k}^{(1,3 a)}$ to the opportunistic non-water-filling solution in (25) with $f_{k}$ in 27), and for all $k, j \in \mathcal{K}, k \neq j$, $(k, m)=(1, d),(2, r),(l, n)=(1,3 a)$. The optimal $P_{r}^{(1,3 a)}$ is given by (14) with the first term scaled by $\alpha$.

Case 2 and Case 3a: The sum-rate optimization for this case is

$$
\max _{P \in \mathcal{B}_{2,3 a}}\left(R_{\mathcal{K}}\right)_{r} \text { s.t. } R_{2, r}^{\min }=R_{2, d}^{\max }
$$


where $\mathcal{B}_{2,3 a}$ is the set of all $\underline{P}$ that satisfy $(18),(28)$, and the remaining inequality in (16). The resulting KKT conditions in (21) use $f_{k}, k=1,2$, defined in (27) but with $(k, m)=(1, r),(2, d)$. Note that $\alpha$ captures the equality condition in (28). For $\left|h_{m, 1}\right| / \nu_{1} \neq\left|h_{m, 2}\right| / \nu_{2}, m=r$ or $d$, and $(k, m)=(1, r),(2, d), P_{k}^{(2,3 a)}$ at each source is given by the opportunistic policy in (25). The optimal relay policy $P_{r}^{(2,3 a)}$ is the same as that obtained in case 1 and case $3 a$.

Case 1 and Case 3b: The sum-rate optimization for this case is

$$
\max _{P \in \mathcal{B}_{1,3 b}}\left(R_{\mathcal{K}}\right)_{d} \text { s.t. } R_{2, d}^{\min }=R_{2, r}^{\max }
$$

where $\mathcal{B}_{1,3 b}$ is the set of all $\underline{P}$ that satisfy (19), (29), and the remaining inequality in (12). The resulting KKT conditions satisfy (21) where $f_{k}, k=1,2$, is given by (27) with the subscript ' $r$ ' replaced by ' $d$ '. Note that $\alpha$ captures the boundary condition in (29). For $\left|h_{m, 1}\right| / \nu_{1} \neq\left|h_{m, 2}\right| / \nu_{2}, m=$ $r$ or $d$, and $(k, m)=(2, r),(1, d), P_{k}^{(1,3 b)}$ at each source is given by the opportunistic non-water-filling solution in (25). The optimal relay policy $P_{r}^{(1,3 b)}$ simplifies to the water-filling solution in 14.

Case 2 and Case $3 b$ : The sum-rate optimization for this case simplifies to

$$
\max _{P \in \mathcal{B}_{2,3 b}}\left(R_{\mathcal{K}}\right)_{d} \text { s.t. } R_{1, d}^{\min }=R_{1, r}^{\max }
$$

where $\mathcal{B}_{2,3 b}$ is the set of all $\underline{P}$ that satisfy (19), (30), and the remaining inequality in (16). We remark that the KKT conditions are the same as (21) where $f_{k}, k=1,2$, is given by (27) with ' $r$ ' replaced by ' $d$ ' and $(k, m)=(1, r),(2, d)$. The resulting $P_{k}^{(2,3 b)}, k=1,2$ are given by the opportunistic policies in (25). Finally, the optimal $P_{r}^{(2,3 b)}$ is the same as that obtained in case 1 and case $3 b$.

Case 1 and Case $3 c$ : The sum-rate optimization for this case is

$$
\max _{P \in \mathcal{B}_{1,3 c}}\left(R_{\mathcal{K}}\right)_{r} \text { s.t. } R_{2, d}^{\min }=R_{2, r}^{\max } \text { and } R_{1, d}^{\max }=R_{1, r}^{\min }
$$

where $\mathcal{B}_{1,3 c}$ is the set of all $\underline{P}$ that satisfy (20), 31), and the remaining inequality in (12). The resulting KKT conditions satisfy (21) for $k=1,2$, and $(k, m)=(1, d),(2, r)$ where

$$
f_{k}=\frac{\alpha_{3}\left|h_{r, k}\right|^{2}}{1+\sum_{j=1}^{2} \frac{\left|h_{r, j}\right|^{2} P_{j}}{\theta}}+\frac{\alpha_{2}\left|h_{d, k}\right|^{2}}{1+\sum_{j=1}^{2} \frac{\left|h_{d, j}\right|^{2} P_{j}}{\theta}}+\frac{\alpha_{1}\left|h_{m, k}\right|^{2}}{1+\frac{\left|h_{m, k}\right|^{2} P_{k}}{\theta}}
$$

and $\alpha_{1}, \alpha_{2}$, and $\alpha_{3}=1-\alpha_{1}-\alpha_{2}$ are Lagrange multipliers that capture the boundary conditions in 31 . When $\left|h_{m, 1}\right| / \nu_{1}$ $\neq\left|h_{m, 2}\right| / \nu_{2}, m=r$ or $d$, for all $k, j \in \mathcal{K}, k \neq j$, and pairs $(k, m)=(1, d),(2, r)$, the optimal $P_{k}^{(1,3 c)}, k=1,2$, is given by the opportunistic policy in (25) while $P_{r}^{(1,3 c)}$ is given by the water-filling solution in (14) with the first term scaled by $\left(\alpha_{1}+\alpha_{2}\right)$.

Case 2 and Case $3 c$ : The sum-rate optimization for this case is

$$
\max _{P \in \mathcal{B}_{2,3 c}}\left(R_{\mathcal{K}}\right)_{r} \text { s.t. } R_{2, d}^{\max }=R_{2, r}^{\min } \text { and } R_{1, d}^{\min }=R_{1, r}^{\max }
$$

where $\mathcal{B}_{2,3 c}$ is the set of all $\underline{P}$ that satisfy $(20),(33)$, and the remaining inequality in (16). The resulting KKT conditions and optimal policy $\underline{P}^{(2,3 c)}$ are the same as in case 1 and case $3 c$ but with $(k, m)=(1, r),(2, d)$.

Finally, the optimal $\underline{P}^{*}$ is given by the following theorem.

Theorem 3: The $\underline{P}^{*}$ that maximizes the sum-rate is obtained by computing $\underline{P}^{(m)}$ or $\underline{P}^{(j, k)}$ starting from case 1 and proceeding one case at a time, until for some case the corresponding $\underline{P}^{(m)}$ or $\underline{P}^{(j, k)}$ satisfies the case conditions.

\section{SUMmARY AND FUTURE WORK}

We have developed the power policy that maximizes the sum-rate of a two-user orthogonal MARC. We have shown that the optimal policy is a function of the channel statistics and network geometry and can be classified into two broad categories. The first category involves cases where each user is clustered with a different receiver as a result of which the sum-rate decouples into independent terms for each user and the relay. The second category includes the cases where the two users are clustered with one of the receivers as well as the boundary cases. The first category admits the classic waterfilling solution at each user and the relay. The optimal policies for the second category do not always result in a water-filling solution at the sources; however, they reveal the optimality of exploiting the multiuser fading diversity to opportunistically schedule users. Our results can be generalized to a $K$-user orthogonal MARC with $K>2$. Finally, one could also consider the resource allocation problem for CF where the challenge lies in solving a non-convex optimization problem.

\section{REFERENCES}

[1] G. Kramer and A. J. van Wijngaarden, "On the white Gaussian multipleacess relay channel," in Proc. 2000 IEEE Int. Symp. Inform. Theory, Sorrento, Italy, June 2000, p. 40.

[2] Y. Liang and V. Veeravalli, "Resource allocation for wireless relay channels," in Proc. 38th Annual Asilomar Conf. Signals, Systems, and Computers, Pacific Grove, CA, Nov. 2004.

[3] L. Sankaranarayanan, G. Kramer, and N. B. Mandayam, "Hierarchical sensor networks: Capacity theorems and cooperative strategies using the multiple-access relay channel model," in Proc. First IEEE Conference on Sensor and Ad Hoc Communications and Networks, Santa Clara, CA, Oct. 2004.

[4] T. Cover and A. El Gamal, "Capacity theorems for the relay channel," IEEE Trans. Inform. Theory, vol. 25, no. 5, pp. 572-584, Sept. 1979.

[5] G. Kramer, M. Gastpar, and P. Gupta, "Cooperative strategies and capacity theorems for relay networks," IEEE Trans. Inform. Theory, vol. 51, no. 9, pp. 3027-3063, Sept. 2005.

[6] L. Sankaranarayanan, G. Kramer, and N. B. Mandayam, "Offset encoding for multiaccess relay channels," Aug. 2006, submitted to the IEEE Trans. Inform. Theory.

[7] A. Host-Madsen and J. Zhang, "Capacity bounds and power allocation for the wireless relay channel," IEEE Trans. Inform. Theory, vol. 51, no. 6, pp. 2020-2040, June 2005.

[8] R. Knopp and P. Humblet, "Information capacity and power control in single-cell multiuser communications," in Proc. IEEE Intl. Conf. Commun., Seattle, WA, June 1995.

[9] D. N. C. Tse and S. V. Hanly, "Multiaccess fading channels - part I: Polymatroid structure, optimal resource allocation and throughput capacities," IEEE Trans. Inform. Theory, vol. 44, no. 7, pp. 2796-2815, Nov. 1998.

[10] S. Boyd and L. Vandenberghe, Convex Optimization. Cambridge, UK: Cambridge University Press, 2004. 